Archives

18-19 | 1997

Inventaire des archives de Robert Mandrou

\title{
Préface à la traduction allemande des Fugger. Version française
}

\section{Étienne François}

\section{(C) OpenEdition}

\section{Journals}

Édition électronique

URL : http://journals.openedition.org/ccrh/2568

DOI : $10.4000 /$ ccrh.2568

ISSN : 1760-7906

Éditeur

Centre de recherches historiques - EHESS

Édition imprimée

Date de publication : 10 octobre 1997

ISSN : 0990-9141

Référence électronique

Étienne François, "Préface à la traduction allemande des Fugger. Version française », Les Cahiers du Centre de Recherches Historiques [En ligne], 18-19 | 1997, mis en ligne le 20 février 2009, consulté le 26 avril 2019. URL : http://journals.openedition.org/ccrh/2568; DOI : 10.4000/ccrh.2568

Ce document a été généré automatiquement le 26 avril 2019

Article L.111-1 du Code de la propriété intellectuelle. 


\title{
Préface à la traduction allemande des Fugger. Version française
}

\author{
Étienne François
}

\section{NOTE DE L'AUTEUR}

Le présent texte représente la version française, légèrement remaniée et mise à jour bibliographiquement, de la préface allemande à la traduction des Fugger : Robert Mandrou, Die Fugger, Adeliger Grundbesitz in Schwaben 1560-1618 (aus dem Französischen übersetzt von Eckart Bimstiel), Göttingen, 1997 (« Veröffentlichungen des Max-Planck Instituts für Geschichte », 136).

1 Six ans après la parution en France des Fugger, l'historien allemand Pankraz Fried, premier titulaire de la chaire d'histoire régionale de l'université récemment créée d'Augsbourg, constatait avec regret dans sa leçon inaugurale, le 9 décembre 1975 : «Ce livre, malheureusement, n'a toujours pas fait l'objet de la traduction allemande qui s'impose, si bien qu'il n'a même pas encore trouvé l'accès aux bibliographies spécialisées de manuels scientifiques $»^{1}$.

2 Cette lacune est enfin comblée, grâce aux efforts conjoints de la Société de recherches sur l'histoire de la Souabe (Schwäbische Forschungsgemeinschaft), de l'Institut Max-Planck d'histoire de Göttingen et de la Mission historique française en Allemagne, grâce aussi à la traduction claire et précise d'Eckart Birnstiel. Mais il aura fallu attendre plus de vingt ans pour que le vœu de Pankraz Fried soit enfin exaucé. Ce délai exceptionnellement long - puisqu'il s'est écoulé près de trente ans entre la parution française et la traduction allemande d'une étude pourtant consacrée à un thème essentiel de l'histoire allemande et saluée dès sa parution comme un travail de première importance - jette un jour particulièrement cru sur la profondeur et la persistance des cloisonnements entre les recherches historiques de nos deux pays. Il incite à relativiser l'impact réel de l'internationalisation croissante de la recherche et la multiplication - à laquelle Robert Mandrou a contribué de manière décisive - des contacts et des échanges entre historiens 
français et allemands. Mais il invite aussi à s'interroger sur les raisons de la méconnaissance persistante d'un grand livre d'histoire, à rappeler la richesse des apports de Robert Mandrou à la recherche historique et l'importance de son rôle de médiateur, à souligner, enfin, l'extraordinaire qualité d'une œuvre dont le temps passé n'a fait que rendre plus évidentes l'originalité et la nouveauté.

La non-réception des Fugger en Allemagne me paraît tenir à trois raisons, elles-mêmes très directement liées à ce qui fait leur originalité. La première tient au fait qu'à l'époque de sa parution ce livre correspondait à un type d'enquête, à un mode d'écriture et à des préoccupations qui étaient largement étrangers à ce qui se pratiquait alors en Allemagne. Située dans le prolongement d'une tradition déjà longue d'histoire sociale du monde des campagnes, nourrie de la lecture et de la confrontation avec les grandes thèses de Gaston Roupnel, de Pierre de Saint-Jacob ou de Pierre Goubert ${ }^{2}$, mais aussi de Boris Porchnev - que Robert Mandrou avait précisément fait connaître et découvrir en France ${ }^{3}$.-, l'enquête sur les Fugger est en effet l'exemple même de ce que François Furet allait appeler quelques années plus tard l'« histoire-problème », c'est-à-dire ce type d'enquête qui donne la priorité absolue à un problème à résoudre, qui commence d'abord par se constituer son objet et qui ensuite - mais ensuite seulement - se met en quête du site et des sources qui seront les mieux à même d'aider à y répondre, tout en multipliant les comparaisons permettant d'échapper au danger du repli monographique ${ }^{4}$. En d'autres termes, si Robert Mandrou choisit de travailler sur les acquisitions foncières et les achats de seigneuries des Fugger, ce n'est pas d'abord pour éclairer un aspect mal connu de l'histoire sociale de l'Allemagne moderne, mais bien plutôt parce que l'exemple des Fugger, l'abondance et la richesse de leurs archives, lui paraissent se prêter mieux que tout à l'examen d'une question qui était alors au centre des débats des historiens français, celle d'une éventuelle "féodalisation" de la bourgeoisie marchande dans l'Europe moderne.

4 La deuxième raison tient au fait que l'enquête de Robert Mandrou est une illustration parfaite du type d'histoire promu par les historiens réunis alors autour de la revue Annales et se réclamant d'elle - ce qui, au reste, ne saurait tout à fait surprendre si l'on se rappelle qu'il fut, comme l'écrivait Pierre Goubert, « le dernier disciple de Lucien Febvre donc l'un des plus chers ${ }^{5}$, qui lui succéda à la sixième section de l'École des hautes études sur la chaire d'histoire sociale des mentalités modernes, et qui fut pendant huit ans, de 1954 à 1962, secrétaire de la revue. Cette enquête, en effet, répond en tous points aux traits caractéristiques retenus par l'historien américain Georg Iggers pour définir la production historique des Annales à cette époque, et dont on chercherait vainement l'équivalent dans la production historique allemande d'alors ${ }^{6}$. Elle se caractérise d'abord par le fait qu'elle "porte sur l'époque moderne ", c'est-à-dire précisément sur l'époque de prédilection des historiens des Annales, par le fait, ensuite, qu'elle se définit comme une histoire des mentalités - entendues comme des "pratiques et représentations plus diffuses que les idées et qui, à la différence de ces dernières qui sont le produit de la pensée d'individualités, sont, elles, la propriété d'un groupe collectif $»^{7}-$, par son "étroite combinaison de l'histoire avec la géographie, l'économie et l'anthropologie ", mais aussi par sa «fascination des chiffres " (pas moins de treize cartes, huit graphiques et vingt-trois tableaux statistiques), par la manière, enfin, dont elle « fait aller de pair histoire sérielle et histoire des mentalités ».

5 La dernière raison tient, enfin, au fait que l'enquête de Robert Mandrou représente la première véritable tentative d'application à un cas allemand de problématiques et de 
méthodes d'investigation forgées pour l'essentiel par des historiens français et jusque-là appliquées presque exclusivement à des cas français. Or cette tentative - dont la traduction enfin offerte au public allemand montre d'évidence à quel point elle fut une réussite - a été opérée à l'époque précisément où les réserves des historiens allemands à l'égard des Annales étaient particulièrement fortes et où les plus novateurs d'entre eux - l'article de Dieter Groh paru deux ans seulement plus tard en est un bon témoignage ${ }^{8}$ se disaient convaincus de l'impossibilité d'appliquer à l'histoire allemande des méthodes et des problématiques trop spécifiquement françaises. Si bien qu'on en est conduit à se demander si la non-réception des Fugger en Allemagne n'est pas due tout simplement au fait que ce livre apportait un démenti flagrant à l'opinion, communément admise par les historiens allemands de l'époque, de l'inadaptation intrinsèque des paradigmes des Annales aux spécificités de l'histoire allemande.

6 La traduction en allemand de ce livre aussi singulier qu'exemplaire s'imposait d'autant plus qu'il opère la synthèse des deux préoccupations majeures de Robert Mandrou : d'un côté, son intérêt pour l'histoire des mentalités, et, de l'autre, les dimensions européennes de ses curiosités et de ses engagements. "Étude de comportements socioéconomiques " guidée par la conviction que "les actes sont plus significatifs que les déclarations d'intention" - pour reprendre les termes mêmes du titre du livre et de son introduction-, l'enquête sur les Fugger est d'abord et avant tout un exemple particulièrement convaincant de cette histoire des mentalités dont Robert Mandrou a plus que tout autre contribué à définir la notion et les méthodes. De ce point de vue, elle s'inscrit dans la continuité de ses autres livres sur l'histoire de la civilisation française du $\mathrm{XVII}^{\mathrm{e}}$ au Xx $\mathrm{x}^{\mathrm{e}}$ siècle, sur les mentalités des différents groupes sociaux de la France moderne et leur évolution, sur la Bibliothèque bleue de Troyes et les problèmes de la lecture et de la culture populaire en France aux XVII et XVIII ${ }^{e}$ siècles, sur les classes et les luttes de classes en France au début du XVII ${ }^{\mathrm{e}}$ siècle, sur l'attitude des magistrats français à l'égard de la sorcellerie au XVII ${ }^{e}$ siècle, ou encore sur l'histoire des rapports complexes qui lient aux XVI ${ }^{\mathrm{e}}$ et $\mathrm{XVII}^{\mathrm{e}}$ siècles les intellectuels aux différents milieux sociaux ${ }^{9}$ - livres qui, tous situés à la rencontre entre l'histoire sociale et l'histoire culturelle, s'attachent à reconstituer la sensibilité et les comportements des différents groupes et milieux sociaux, et montrent en même temps que ces groupes, loin d'être enfermés dans leur vision du monde, s'influençaient au contraire mutuellement et ne peuvent être de ce fait compris que dans leurs interactions, leurs échanges et leurs conflits.

7 Étude d'histoire allemande, l'enquête sur les Fugger témoigne par ailleurs de l'ouverture européenne de Robert Mandrou et de l'intérêt constant qu'il porta à l'Allemagne et son histoire - à la différence de la majorité des autres historiens français, soit exclusivement préoccupés par la France, soit tournés de préférence vers le monde méditerranéen. Cette découverte de l'Allemagne fut chez lui précoce et s'opéra dans les pires conditions : en 1943, en effet, alors qu'il était étudiant à Lyon, il fut envoyé de force en Allemagne dans le cadre du « service du travail obligatoire ». Employé d'abord dans une usine de Brunswick, il en fut retiré pour " mauvais esprit ", ayant une influence néfaste sur ses camarades, et envoyé en punition comme bûcheron dans la forêt du Harz. Cette expérience d'épreuve eut une influence déterminante et d'autant plus forte qu'avec le refus du sectarisme, l'indépendance de jugement et l'exigence de lucidité qui le caractérisaient il se refusa de confondre le nazisme et la culture allemande. L'intérêt qu'il en retira pour la civilisation et l'histoire du monde germanique et de l'Europe centrale marqua, en effet, toute son activité. En témoignent - outre l'enquête sur les Fugger - ses nombreux comptes rendus 
de livres allemands (en particulier à l'époque où il était secrétaire des Annales), les thèmes abordés dans le cadre de son séminaire ${ }^{10}$, les treize articles qu'il publia en allemand ou dans une revue allemande, ses interventions en Allemagne même pour mieux faire connaître les nouvelles directions de la recherche historique française ${ }^{11}$, son dernier grand livre Staatsräson und Vernunft, 1649-1775, paru d'abord en allemand (dans l'élégante traduction de Michael Erbe) en 1976 dans la prestigieuse collection de la «Propyläen Geschichte Europas ", avant d'être publié en France et en français ${ }^{12}$, et, enfin, son dernier projet, la création en 1977, à force de ténacité et alors même qu'il était déjà atteint par la maladie, de la «Mission historique française en Allemagne" implantée à Göttingen, auprès de l'Institut Max-Planck d'histoire et en symbiose avec lui, et dont il voulait faire un lieu de rencontre et de recherche, dans un climat de confrontation critique et d'ouverture européenne. Car l'intérêt prioritaire porté par Robert Mandrou à l'espace germanique ne prenait lui-même tout son sens que dans une dimension européenne : il suffit pour s'en rendre compte de regarder, dans le volume de Mélanges qui lui a été consacré, la carte de ses missions, congrès, colloques et conférences à l'étranger entre 1960 et $1978^{13}$, de constater l'ampleur et l'ouverture des lectures qui ont accompagné son enquête sur les Fugger ${ }^{14}$, ou, encore, d'évoquer son engagement pour la Tchécoslovaquie, victime de la répression et de la normalisation ${ }^{15}$.

Pour regrettable qu'elle soit, la date tardive de la traduction en allemand des Fugger n'en a pas moins un mérite : celui de mieux mettre en lumière le caractère foncièrement novateur et exemplaire d'une œuvre qui reste toujours aussi singulière - l'extrême rareté des recherches menées en Allemagne sur les rapports entre villes et campagnes - à l'exception de la remarquable étude de Rolf Kiessling qui porte, elle aussi (et ce n'est pas un hasard) sur la Souabe du $\mathrm{XIV}^{\mathrm{e}}$ au $\mathrm{XVI}^{\mathrm{e}}$ siècle - en est la meilleure preuve ${ }^{16}$. Son exemplarité et son extraordinaire résistance à l'usure du temps, elle les doit, d'abord, au soin extrême de son écriture : tout entière centrée sur la démonstration, servie par une langue classique et épurée, dense et claire, qui pèse les moindres nuances, elle constitue, de ce point de vue, "un modèle d'écriture historique " $\left(\mathrm{Ph}\right.$. Joutard ${ }^{17}$ ). Elle les doit, ensuite, à la multiplicité des perspectives qu'elle ouvre, depuis celles que Robert Mandrou évoque lui-même et qu'il aurait aimé reprendre - telles l'exploitation de séries longues de comptes des intendants des Fugger après $1650^{18}$, l'analyse micro-historique de l'endettement paysan ${ }^{19}$, ou encore la reconstitution de la "condition paysanne sur les seigneuries des Fugger $»^{20}-$, jusqu'à celles que suggère la lecture de son livre, telle l'analyse des éléments d'innovation subreptices introduits par la gestion des Fugger (défrichements, endiguements, introduction de nouvelles races bovines) ${ }^{21} \mathrm{ou}$, encore, l'analyse des rapports féodaux des Fugger avec les autres détenteurs de seigneuries de la région. Elle les doit, enfin, au fait qu'elle paraît anticiper à vingt-cinq ans de distance certaines des tendances majeures du renouvellement contemporain de la recherche historique - qu'il s'agisse de l'approche des comportements socio-économiques dans une perspective d'anthropologie sociale et culturelle, du recours à l'analyse micro-historique ou de l'usage du comparatisme en tant que confrontation critique, en vue d'une confrontation réciproque, des approches et des méthodes, des traditions historiographiques et des styles d'écriture (parmi les projets non réalisés de Robert Mandrou figure aussi celui d'une étude comparée de la condition paysanne en Souabe et en Bohême à la veille de la guerre de Trente Ans).

Pour un livre d'histoire comme pour tout autre livre, les deux épreuves les plus redoutables sont celles du temps et de la traduction. L'exceptionnelle aisance avec 
laquelle les Fugger les ont surmontées prouve d'évidence la fécondité persistante et l'exemplarité d'un historien trop tôt disparu qui a joué un rôle essentiel, bien que souvent méconnu, dans le renouvellement des approches historiographiques, qui fut un remarquable médiateur entre la France et l'Allemagne, et dont la vie comme l'œuvre attestent que la vitalité de la recherche historique passe aussi bien par l'ouverture intellectuelle et l'exigence d'écriture, que par le dépassement des cloisonnements disciplinaires et des frontières nationales.

\section{NOTES}

1. Pankraz Fried, Die Fugger in der Herrschaftsgeschichte Schwabens, Munich, 1982, note 4, p. 6 («Schriften der Philosophischen Fachbereiche der Universität Augsburg », n 9).

2. Outre le modèle ancien, mais combien important pour Robert Mandrou, représenté par la thèse de Lucien Febvre, Philippe II et la Franche-Comté, étude d'histoire politique, religieuse et sociale, Paris, 1911, ont compté particulièrement pour lui les thèses de Gaston Roupnel, La Ville et la campagne au XVII siècle, étude sur la population du pays dijonnais, Paris, 1955 ; Pierre de Saint-Jacob, Les Paysans de la Bourgogne du Nord au dernier siècle de l'Ancien Régime, Paris 1960 ; et Pierre Goubert, Beauvais et le Beauvaisis de 1600 à 1730. Contribution à l'histoire sociale de la France au XVII siècle, Paris, 1960.

3. Après avoir rendu compte du livre de B. Porchnev qu'il avait lu dans sa version allemande - " Les soulèvements populaires et la société française au XVII ${ }^{\mathrm{e}}$ siècle », Annales ESC, XIV, 1959, p. 756-765 - Robert Mandrou se chargea d'en faire éditer une traduction française, parue quatre ans seulement plus tard sous le titre : Boris Porchnev, Les Soulèvements populaires en France de 1623 à 1648, collection « CEuvres étrangères » du Centre de recherches historiques, Paris, 1963.

4. François Furet, « De l'histoire-récit à l'histoire-problème », Diogène, 89, 1975.

5. Cette expression figure dans la chronique nécrologique que Pierre Goubert fit paraitre sous le titre « La séduction et l'intelligence » dans le journal Le Monde du $1^{\mathrm{er}}-2$ avril 1984. Cette chronique est reproduite en hors-texte dans l'émouvant volume publié par les soins de Philippe Joutard et Jean Lecuir, Histoire sociale, sensibilités collectives et mentalités, Mélanges Robert Mandrou, Paris, 1985.

6. Citations tirées (en traduction française) du livre de Georg G. Iggers, Geschichtswissenschaft im 20. Jahrhundert, Ein kritischer Überblick im internationalen Vergleich, Göttingen, 1993, p. 49, 48, 43, 47 et 48. Voir également l'étude très détaillée de Lutz Raphael, Die Erben von Bloch und Febvre Annales - Geschichtsschreibung und nouvelle histoire in Frankreich, 1945-1980, Stuttgart, 1994, qui rappelle, elle aussi, que Robert Mandrou était devenu «le collaborateur le plus étroit et le disciple le plus proche» de Lucien Febvre (p. 217), qui rend justice au rôle essentiel joué par Robert Mandrou dans la «constellation " des Annales et de la $\mathrm{VI}^{\mathrm{e}}$ section - son nom est cité aussi souvent que celui de G. Duby, F. Furet ou J. Le Goff, et plus, par exemple, que celui de P. Chaunu, J. Delumeau, M. Ferro ou P. Goubert - et souligne l'importance et la nouveauté des directions et des impulsions données à la revue pendant les années où il en fut l'âme (histoire des mentalités, ouverture européenne).

7. Voir deux phrases de l'introduction où Robert Mandrou parle de son essai « d'appréhender à l'aide des comptes tenus par les Fugger, pour leurs biens fonciers en Souabe, la réalité de leurs 
comportements en ce domaine » (p. 15), car « les actes sont plus significatifs que les déclarations d'intention » (p. 13).

8. Dieter Groh, "Strukturgeschichte als totale Geschichte", Vierteljahrsschrift für Sozial-und Wirtschaftsgeschichte, 58, 1971, p. 289-322. Sur les différences entre l'historiographie française et l'historiographie allemande des années soixante, voir, outre l'article de Hartmut Kaelble, «L'histoire sociale en France et en Allemagne fédérale: de l'ignorance cordiale aux promesses d'un nouveau dialogue ", Bulletin d'information de la Mission historique française en Allemagne, 12, 1986, p. 9-28, et l'essai critique et stimulant sur les blocages persistants de l'historiographie allemande à l'égard des Annales et leurs (possibles et probables) raisons, de Peter Schöttler, « Zur Geschichte der Annales - Rezeption in Deutschland (West) », Matthias Middell et Steffen Sammler (Dir.), Alles Gewordene hat Geschichte. Die Schule des Annales in ihren Texten, 1929-1992, Leipzig, 1994, p. 40-60, ainsi que les pages 485-497 de l'ouvrage précédemment évoqué de Lutz Raphael.

9. Robert Mandrou, Histoire de la civilisation française (avec Georges Duby), t. I, Moyen Âge-XVI siècle, t. II, XVII $-\mathrm{XX}$ siècle, Paris, 1958; Introduction à la France moderne (1500-1640). Essai de psychologie historique, Paris, 1961; De la culture populaire en France aux XVII et XVIII ${ }^{e}$ siècles. La Bibliothèque bleue de Troyes, Paris, 1964 ; Classes et luttes de classes en France au début du XVII siècle, Florence, 1965 ; Magistrats et sorciers en France au XVII siècle. Une analyse de psychologie historique, Paris 1968 ; Des humanistes aux hommes de science (XVI ${ }^{e}$ et XVII ${ }^{e}$ siècles), Paris, 1973.

10. De 1963 à 1966, son séminaire porta sur «mentalités et économie rurales en Souabe à la fin du XVI siècle»; en 1973-1974, il fut consacré aux "échanges intellectuels entre France et Allemagne au XVII ${ }^{\mathrm{e}}$ siècle : le duc August de Brunswick-Wolfenbüttel ». L. Raphael, op. cit., p. 605 (annexe C), programme du séminaire d'histoire des mentalités modernes animé par Robert Mandrou de 1959 à 1975.

11. À titre d'illustration, on évoquera son article "Probleme und Methoden der heutigen französischen Geschichtsforschung », Jahrbücher für Nationalökonomie und Statistik, 176, 1974, p. 359-370, ainsi que son intervention sur «culture nobiliaire et culture populaire en France » dans le cadre du congrès des historiens allemands «Historikertag" de Ratisbonne en 1972, publiée deux ans plus tard dans la principale revue historique allemande: "Adelskultur und Volkskultur in Frankreich », Historische Zeitschrift, 217, 1974, p. 36-53.

12. Staatsräson und Vernunft, 1649-1775, Francfort-Berlin-Vienne, 1976 («Propyläen Geschichte Europas », t. III) ; L'Europe absolutiste. Raison et raison d'Etat, 1649-1775, Paris, 1977. Le traducteur en allemand de ce livre, alors enseignant d'histoire à l'université libre de Berlin, est également l'auteur d'une des meilleures présentations en allemand des Annales et du groupe d'historiens s'en réclamant: Michael Erbe, Zur neueren französischen Sozialgeschichtsforschung. Die Gruppe um die Annales, Darmstadt, 1979. Lors de la parution en Allemagne de Staatsräson und Vernunft, l'historien Golo Mann (un des fils de Thomas Mann, auteur, entre autres choses, d'une extraordinaire biographie de Wallenstein) consacra à ce livre une pleine page d'éloges dithyrambiques dans l'hebdomadaire Die Zeit (17 septembre 1976, nº 39, p. 25-26).

13. Histoire sociale, sensibilités collectives et mentalités, op. cit., p. 17.

14. À côté des références en provenance de France ou d'Europe occidentale, on relève dans sa bibliographie des ouvrages d'historiens est-allemands, hongrois, polonais, autrichiens, russes et slovaques.

15. À la suite de l'invasion de la Tchécoslovaquie par les troupes du pacte de Varsovie, Robert Mandrou assura la publication en France de Les Sept jours de Prague, 21-27 août 1968. Première documentation historique complète de l'entrée des troupes aux accords de Moscou, Paris, 1969. Il persuada par ailleurs son ami Josef Macek d'écrire à l'intention du public français une histoire de Bohême qui parut, avec une préface de lui, peu après sa mort: Josef Macek, Histoire de la Bohême des origines à 1918, Paris, 1984. 
16. Rolf Kiessling, Die Stadt und ihr Land. Umlandpolitik, Bürgerbesit und Wirtschaftsgefüge in Ostschwaben vom 14. bis zum 16. Jahrhundert, Cologne-Vienne, 1989 («Städteforschung », Reihe A : Darstellungen, vol. 29).

17. Philippe Joutard, "L'itinéraire d'un historien européen au $\mathrm{xx}^{\mathrm{e}}$ siècle ", Histoire sociale, sensibilités collectives et mentalités, op. cit., p. 19.

18. Dans la note 13 de la page 19, Robert Mandrou évoque son souhait d'une exploitation des séries longues de comptes des intendants, mentionnant en particulier les séries de Kirchberg (1650-1804), Kirchheim (1598-1804) ou Nordendorf (1581-1804).

19. Cf. note 10, p. 208.

20. Cf. note 8, p. 95 .

21. Cf. la remarque de la note 77, p. 134 . 